\title{
Myeloid Neoplasms with Germline Predisposition Associated with Other Organ Dysfunction
}

National Cancer Institute

\section{Source}

National Cancer Institute. Myeloid Neoplasms with Germline Predisposition Associated

with Other Organ Dysfunction. NCI Thesaurus. Code C151910.

Familial myeloid neoplasms associated with germline mutations and a preexisting disorder or organ dysfunction other than a platelet disorder. 